\title{
CAMBIOS EN EL CONTENIDO DE OXITRIMETILAMINA (OTMA), TRIMETILAMINA (TMA) Y NITRÓGENO VOLÁTIL TOTAL (TVN) EN EL BACALO DE PROFUNDIDAD (Dissostichus eleginoides Smith), EL LENGUADO COMÚN (Paralichthys adspersus) YEL SARGO (Anisotremus scapularis) ALMACENADOS EN HIELO
}

Quitero Valencia Mecola ${ }^{\prime}$, Leonardo Sheron Ramirez ${ }^{2}$ Reyna Calcino Angulo ${ }^{3}$

\section{RESUMEN}

Los autores han hecho la determinación del contenido de Oxitrimetilamina, trimetilamina y nitrógeno total volatil en el músculo del bacalo de profundidad (Dissotichus eleginiodes), del lenguado común (Paralichthy adspersus) y del sargo (Anisotremus scapulareis) almacenados en hielo. Estas especies fueron capturadas entre Atico y Tacna: el trabajo tuvo como objetivo verificar la relación existente entre el contenido de OTMA, TMA Y TVN en el músculo del pescado y establecer la utilidad de los indicadores elegidos para establecer la calidad del pescado almacenado.

Se ha podido establecer que durante el almacenamiento en hielo de las tres diferentes especies, difieren entre sí, aunque los cambios son similares en el caso del bacalao y del lenguado; no así en el del sargo en estas mismas condiciones.

Hay una estrecha relación entre el aumento del TMA y el empeoramiento de las cualidades organolipticas durante el almacenamiento del pescado, las cuales se manifiestan según la especie en el bacalao fue de 4,18-15,55 $\mathrm{mg} \mathrm{N} / 100 \mathrm{~g}$ ) entre el noveno y decimo día, para el lenguado entre el décimo y onceavo día (de 2,19 a $4,26 \mathrm{mg} \mathrm{N} /$ $100 \mathrm{~g}$, para el sargo entre el sexto y séptimo día (de 0,85-1,15 $\mathrm{mg} \mathrm{N} / 100 \mathrm{~g}$.

\section{A B S TR ACT}

The Authors have made the determination of the content of Oxitrimetilamina, trimetilamina and volatile total nitrogen in the muscle of the bacalo of depth (Dissotichus eleginiodes), of the common sole (Paralichthy adspersus) and of the sargo (Anisotremus scapulareis) stored in ice. These species were captured between Loft and Tacna: The work had as objective to verify the existent relationship among the content of OTMA, TMA AND TVN in the muscle of the fish and to establish the utility of the elected indicators to establish the quality of the stored fish.

It can have been establih that during he tnee different species storcing in ice process, they differ one to each other, even changes are similar in case of the cod and the sole, except in Sargo under these conditions.

There is a narrow relationship between the increment of TMA and the worsening of organoliptic qualities during the storing, which are manifested according to the species, it was 4,18-15,55 $\mathrm{mg} \mathrm{N} / 100 \mathrm{~g}$ in cod between the 9 th to 10 th day, $2,19-4,26 \mathrm{mg} \mathrm{N} / 100 \mathrm{~g}$ in sole between the 10 th and 11 th day and 0,85-1,15 $\mathrm{mg} \mathrm{N} / 100 \mathrm{~g}$ for the sargo between the sixth and seventh day. 


\section{INTRODUCCIÓN}

Desde mucho tiempo atrás la oxitrimetilamina (OTMA), la trimetilamina (TMA) y el nitrógeno volatil total (TVN) son los indicadores más comunes que se utilizan para la determinación de la frescura y calidad del pescado. Dichas sustancias se presentan después de la muerte del pescado. Inicialmente estos componentes están en pequeñas cantidades, de tal modo que conforme va transcurrir el tiempo de almacenamiento se van incrementado, to cual depende de la forma como esté almacenado el producto y del tiempo que éste dure (1).

La OTMA se encuentra distribuida en las especies marinas, reportándose un alto contenido en los tejidos de los peces elasmobronquios (500-1500 mg/100g.), en segundo termino también es representativo lo encontrado en el tejido de las especies gadiformes $(2,3)$.

La biosíntesis de la OTMA en el pescado se produce gracias a la unión de la glicinbetaina y colina y TMA (4), En algunas algas marinas se han encontrado altos niveles de OTMA, y es posible la incorporación directa de OTMA mediante la alimentación de los animales acuáticos con estas algas (5).

La TMA es originada por la acción de las bacterias presentes en el pescado, dicha actividad es favorecida por el trabajo de los enzimas de los músculos del pescado, los cuales elaboran el substrato para el desarrollo bacteriano. La TMA es el principal factor que determina el olor a "pescado" (6).

Otro indicador importante de la frescura del pescado es el contenido de nitrógeno voláti total, el que después de algunos dias de almacenamiento en hielo, en las especies gadiformes, se puede encontrar un nivel de $15 \mathrm{mg}$ de nitrógeno en $100 \mathrm{~g}$ de muestra, correspondiendo la mayor parte al amoniaco; a los 15 dias, en algunos casos, se ha podido encontrar hasta $35 \mathrm{mg} \mathrm{N} / 100 \mathrm{~g}$ de muestra (7).

En el presente trabajo se ha considerado el estudio de ios compuestos antes indicados que se pueden presentar durante el almacenamiento, por cuanto hasta hace algunos años atrás, en nuestro país no se reportaban capturas del bacalao de profundidad (Dissotichus eleginoides Smith) (10), pero a partir del año 1996, período durante el cual se llevó a cabo la pesca exploratoria por miembros del Centro de Entrenamiento Pesquero de Paita (8), y por iniciativa privada, se registran algunas capturas que van en ascenso (9). Asimismo, este recurso es muy preciado en el exterior, en mercados como E.E.U.U. a donde se exporta fresco y congelado.

Las dos especies restantes, lenguado común (Paralichthys adspersus) y el Sargo (Anisotremus scapularis) (10), también son de interés comercial tanto a nivel nacional como en el extranjero.

\section{OBJETIVOS}

Los objetivos del presente trabajo:

- Verificar la relación existente entre el contenido de OTMA, TMA y TVN que están en el músculo del pescado durante el periodo de su almacenamiento.

- Establecer la utilidad de los indicadores investigados para determinar la frescura del pescado.

\section{MATERIALES Y MÉTODOS}

\subsection{MATERIAL}

Para la determinación cuantitativa inicial de la OTMA, TMA y TVN, se usó pescado totalmente fresco, recién capturado en la región sur de país.

En el caso del bacalao de profundidad (Dissostichus eleginoides), en la captura se utilizó una embarcación de pesca artesanal de una capacidad de bodega de 10 toneladas, la que se acondicionó previamente, de tal manera que se puedan realizar parte de los análisis respectivos.

Antes de hacerse a la mar la embarcación se cargó con 3 toneladas de hielo machacado y en barras; también se llevó dos cajas isotérmicas de 373 litros de capacidad; también se llevó abordo material de laboratorio, tales como : frascos de vidrio con tapa esmerilada, tubos de vidrio "pesa muestras", molino de carne manual, balones, reactivos, etc. El día 19-07-98, a la altura de Ático ( $16^{\circ} 30.08^{\prime} \mathrm{S}$ y $74^{\circ} 15.2^{\prime} \mathrm{W}$ ), a una profundidad de $1650 \mathrm{~m}$, empleando como aparejo un espinel de fondo de 1200 $m$ de longitud, se inició la faena de pesca comenzando atenderia a las 18:30 horas hasta las 19:15 horas, quedándose toda la noche hasta el día siguiente a las 8:10 horas (dia 20-07-98), hora en la cual se comenzó a recoger el espinel, labor que demoró hasta las 12:30 horas. El número de ejemplares capturados fue 14, su tamaño varió entre 
81 y $98 \mathrm{~cm}$ de largo. Para el estudio se seleccionó un ejemplar de $85,6 \mathrm{~cm}$ de longitud total, su peso fue de $6,5 \mathrm{Kg}$. El espécimen recibido se encontraba en pleno rigor mortis, se observó las cualidades organolépticas y se procedió a eliminar las vísceras y la cabeza, se enjuagó con agua de mar e inmediatamente se colocó dentro de la caja isotérmica con hielo, el cual fue agregado de tal modo que cubriera al animal( $30 \mathrm{Kg}$ aproximadamente). Luego se tomó la primera muestra, la cual consistió en un medallón de $3 \mathrm{~cm}$ de espesor de la parte de la cabeza, se separó la piel y el músculo de la parte ósea y se procedió a moler la carne en el molino, de este modo se obtuvo una pasta, la que sirvió para los análisis respectivos. A Tacna se retornó el día 28 , la muestra de inmediato se trasladó al Laboratorio de Tecnologia Pesquera de la Facultad de Ingeniería Pesquera, UNJBG para proseguir con el trabajo.

En la embarcación se trabajó durante nueve días, que es el tiempo que se permaneció en el mar. Otra operación que se realizó es la reposición del hielo fundido de tal modo que todo el tiempo se mantuvo el pescado enhielado.

Con las especies lenguado y sargo, el trabajo fue similar, solamente que para estos pescados no se salió a bordo por cuanto su captura es costera y diariamente se recibe el pescado, por lo que se contrató a un pescador para que nos abasteciera cada una de las especies, las cuales deberían de venir debidamente enhieladas dentro de una caja isotérmica. Efectivamente, cuando se recibieron los pescados se tuvo cuidado de que el material esté en rigor mortis y con el hielo necesario en la caja isotérmica.

El día 03 de setiembre de 1998 , se realizó la captura del lenguado por inmediaciones de Morro Sama; inmediatamente de haber recibido la muestra se verificó el estado: estaba en pleno rigor mortis. Procediendo a la verificación de su estado, fue trasladado al citado Laboratorio de Tecnología Pesquera de la Facultad de Ingeniería Pesquera, donde se procedió de inmediato a hacer el análisis respectivo.

El día 10 de noviembre de 1998 , se recibió la muestra de sargo; capturado con chinchorro a la altura de Llostay, el tratamiento fue en idénticas condiciones del anterior, procediéndose a realizar el mismo trabajo.

\subsection{METODOS ANÁLITICOS}

El peso de las muestras (después de la captura) se determinó con una balanza manual de fabricación china marca TIABAO, que pesa $0-10 \mathrm{Kg}$. con una precisión de $0,05 \mathrm{Kg}$.

Las muestras para los análisis fueron pesadas, en el caso del bacalao, cuando se estuvo a bordo, en un tubo de vidrio, el cual se preparó especialmente perforándolo por los extremos; las muestras fueron llenadas dentro del tubo después de haber sido molidas; luego se vaciaron a un erlemenyer con tapa esmerilada, con ayuda de un émbolo construido para que no quede muestra; previamente se hizo la verificación del peso en el laboratorio, se pudo comprobar que su capacidad es de $15,1685 \mathrm{~g} \mathrm{t}$ $0,013 \mathrm{~g}$, una vez que la muestra estuviera en el matraz, se le agregó ácido tricloro acético al $10 \%$ y se agitó durante media hora, después se colocó en una caja isotérmica con hielo para transportarlo después al laboratorio. En el laboratorio las muestras se pesaron en una balanza de precisión - OHAUS (0,1 de g. precisión.)

La cantidad de OTMA, TMA se determinó de acuerdo al método Dyer modificado, indicado en el manual de "Métodos Químicos» de la Agencia de Cooperación Internacional del Japón (JICA) (11), el cual consiste en la medición colorimétrica del compuesto coloreado resultante de la reacción de TMA con el ácido pícrico en un lecho de tolueno anhidro. La intensidad de la colocación se determina en un espectro fotómetro marca CAR ZEISS JENA ESPEKOL 210, a una longitud de onda de $410 \mathrm{~nm}$.

La OTM se determina también colorimétricamente, en forma similar que la TMA, solamente en este caso se tuvo que hacer la reducción a TMA, mediante el tricloruro de titanio y nitrato de potasio.

EI TVN se determina mediante la destilación con arrastre de vapor, el cual actúa sobre una porción de extracto de la muestra TCA en presencia de óxido de magnesio $(\mathrm{MgO})$, el destilado se recibe en una solución valorada de $\mathrm{HCl}$ y el exceso se titula con $\mathrm{NaOH}$ también valorado (12). 


\section{RESULTADOS Y DISCUSIÓN}

\subsection{Para el Bacalao de fondo}

Los resultados de las determinaciones del contenido de OTMA, TMA y TVN en los filetes del bacalao de profundidad almacenado en hielo durante 15 dias se presentan en la tabla $N^{\circ} 1$ y en las gráficas número 1,2 y 3 , la cuales se han construido con estos resultados.

Cuadro 1. Contenido de OTMA, TMA y TVN en el bacalao de profundidad almacenado en hielo. (En $\mathrm{mg} N / 100 \mathrm{~g}$ ).

\begin{tabular}{|c|c|c|c|}
\hline $\begin{array}{c}\text { Tiempo de } \\
\text { almacenamiento } \\
\text { (días) }\end{array}$ & OTMA & TMA & TVN \\
\hline 0 & 62,52 & 0,148 & 18,96 \\
1 & 38,53 & 0,176 & 18,83 \\
2 & 60,74 & 0,285 & 18,70 \\
3 & 37,04 & 0,995 & 17,40 \\
4 & 55,79 & 0,945 & 17,80 \\
5 & 49,18 & 1,968 & 17,50 \\
7 & 50,15 & 4,010 & 20,20 \\
8 & 49,52 & 4,020 & 20,60 \\
9 & 43,21 & 4,180 & 19,60 \\
10 & 32,83 & 15,550 & 32,20 \\
11 & 29,75 & 10,160 & 24,20 \\
12 & 27,01 & 14,090 & 24,90 \\
14 & 26,49 & 15,580 & 25,43 \\
15 & 24,87 & 16,220 & 26,80 \\
\hline
\end{tabular}

Durante los siete primeros días de almacenamiento en hielo la variación de los diferentes compuestos fue lenta. El contenido de OTMA decreció de $62,52 \mathrm{mg} \mathrm{N} / 100 \mathrm{~g}$ a $50,15 \mathrm{mg} \mathrm{N} /$ $100 \mathrm{~g}$, la cantidad de TMA aumentó de $0,148 \mathrm{mg} \mathrm{N} /$ $100 \mathrm{~g}$ a $4,010 \mathrm{mg} \mathrm{N} / 100 \mathrm{~g}$ y el TVN aumentó de $18,96 \mathrm{mg} \mathrm{N} / 100 \mathrm{~g}$ a $20,20 \mathrm{~g} \mathrm{~N} / 100 \mathrm{~g}$. Igualmente en forma lenta se fueron notando los cambios organolépticos.

Entre el octavo y décimo día de almacenamiento en hielo la calidad del pescado rápidamente comenzó a decrecer, en ese periodo se puede apreciar la rápida caída de la OTMA hasta $32,83 \mathrm{mg} \mathrm{N} / 100 \mathrm{~g}$; asimismo aumento la TMA y el TVN hasta 4,8 y $19,6 \mathrm{mg} \mathrm{N} / 100$ g. respectivamente. En el onceavo dia de almacenamiento se notó no buenas señales organolépticas, calificándose al pescado no tan bueno para el consumo.

A los quince días la cantidad de OTMA fue de $24,87 \mathrm{mg} \mathrm{N} / 100 \mathrm{~g}$, la TMA $16,22 \mathrm{mg} \mathrm{N} / 100 \mathrm{~g}$; $\sin$ embargo, el TVN fue de $26,80 \mathrm{mg} \mathrm{N} / 100 \mathrm{~g}$.
Observando las Gráficas Nro. 1 y 2 se ve que no hay una relación entre el desarrollo de la curva de TMA en la cual se observa un rápido incremento del contenido y un lento decrecimiento en la OTMA, lo cual puede deberse a efectos secundarios que dan origen a la producción de TMA, posiblemente provenga de la colina, como resultado de la acción de las enzimas de algunos géneros de bacterias presentes en el pescado, lo cual es corroborado por Dyer (citado por Sikorski) (7).

Figura 1. Contenido de OTMA en el Bacalao de Profundidad almacenado en hielo (En mg N/100 g de muestra)

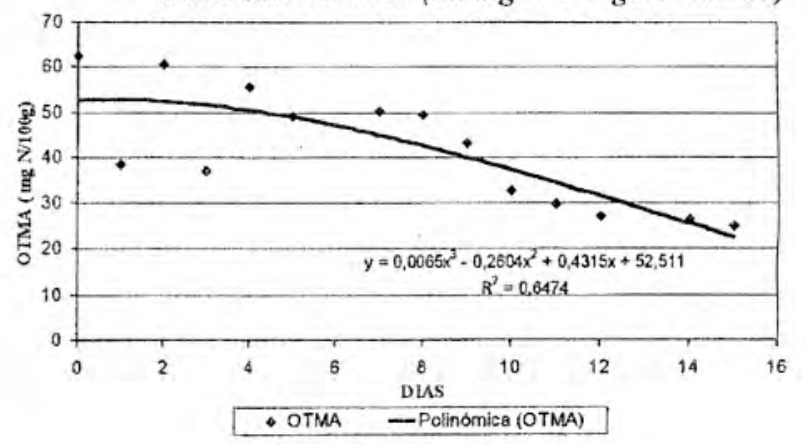

Figura 2. Contenido de TMA en el Bacalao de Profundidad almacenado en hielo (En mg N/100 g de muestra)

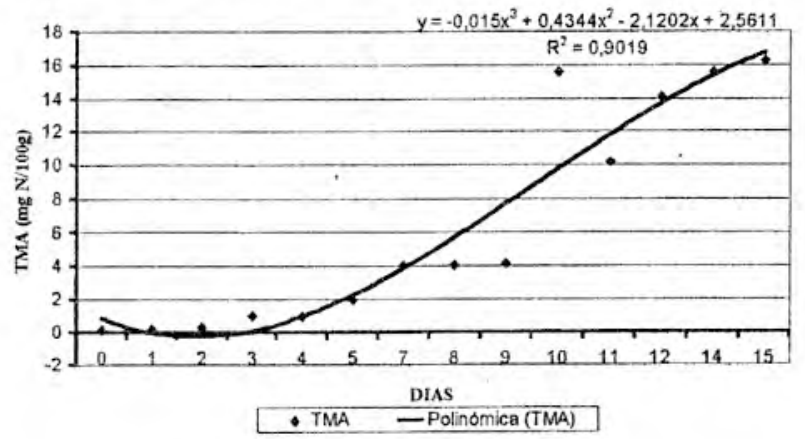

Figura 3. Contenido de TVN en el Bacalao de Profundidad almacenado en hielo (En mg N/100 g de muestra)

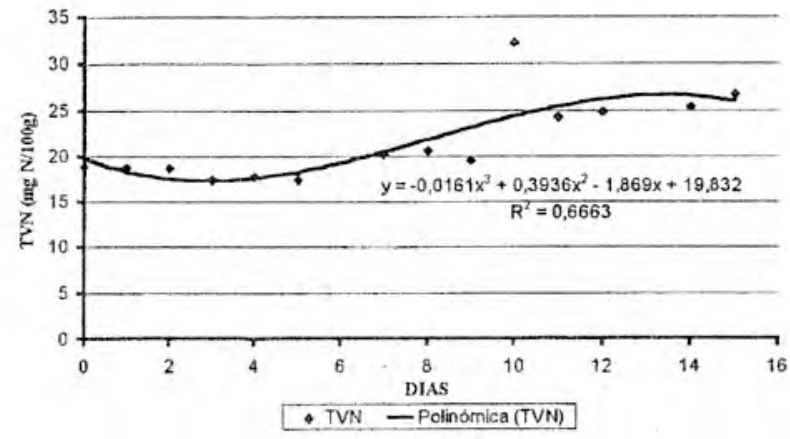




\subsection{Para el lenguado común}

Los resultados de los análisis efectuados para determinar la cantidad de OTMA, TMA y TVN en el pescado fresco almacenado en 15 días en hielo, se presentan en la tabla №2 y en la gráfica también $\mathrm{N}^{\circ} 2$, cuyas curvas se han graficado con estos resultados.

Cuadro 2. Contenido de OTMA, TMA,TVNenel lenguado común fresco almacenado en hielo (En $\mathrm{mg} \mathrm{N} / 100 \mathrm{~g} \mathrm{el}$ muestreo)

\begin{tabular}{|c|c|c|c|}
\hline $\begin{array}{c}\text { Tiempo de } \\
\text { almacenamiento } \\
\text { (días) }\end{array}$ & OTMA & TMA & TVN \\
\hline 0 & 11,06 & 0,15 & 21,84 \\
1 & 14,38 & 0,13 & 21,28 \\
3 & 8,38 & 0,19 & 22,26 \\
4 & 7,20 & 0,27 & 21,84 \\
6 & 13,65 & 0,42 & 19,60 \\
7 & 11,72 & 1,03 & 20,72 \\
8 & 14,93 & 2,01 & 25,34 \\
9 & 13,12 & 2,19 & 22,12 \\
10 & 12,48 & 2,35 & 24,08 \\
11 & 9,81 & 4,26 & 28,60 \\
13 & 2,90 & 2,81 & 22,12 \\
14 & 1,40 & 3,43 & 19,12 \\
15 & 5,26 & 3,75 & 22,12 \\
\hline
\end{tabular}

El contenido del OTMA en el día inicial fue 11,06 $\mathrm{mg} \mathrm{N} / 100 \mathrm{~g}$, de la TMA $0,15 \mathrm{mg} \mathrm{N} / 100 \mathrm{~g}$ y el TVN $21,84 \mathrm{mg} \mathrm{N} / 100 \mathrm{~g}$.

En el período de los 6 primeros días no se verificaron cambios significativos de las cantidades de los compuesto investigados; asimismo, tampoco se observó cambios organolépticos sustanciales. Después del séptimo día de almacenamiento comenzaron a aparecer los primeros cambios organolépticos, la calidad del pescado comenzó a empeorarse. Los valores de cada una de las determinaciones químicas fueron las siguientes: OTMA $11,72 \mathrm{mg} \mathrm{N} / 100 \mathrm{~g}$, TMA $1,03 \mathrm{mg} \mathrm{N} / 100 \mathrm{~g}$ y TVN $20,72 \mathrm{mg} \mathrm{N} / 100 \mathrm{~g}$ al treceavo dia de almacenamiento, de acuerdo con la apreciación organoléptica, se pudo establecer que el pescado no era apto para el consumo. En este día los valores de OTMA decrecieron hasta $2,9 \mathrm{mg} \mathrm{N} / 100 \mathrm{~g}$, TMA aumentó hasta $2,81 \mathrm{mg} \mathrm{N} / 100 \mathrm{~g}$; el valor de TVN fue de 22,12 $\mathrm{mg} \mathrm{N} / 100 \mathrm{~g}$.

Al observar las curvas de las Gráficas 4,5 y 6 se ve que son más tenues en su desarrollo, no tan definidas a diferencia de las desarrolladas en el bacalao.

Figura 4. Contenido de OTMA en el Lenguado de Profundidad almacenado en hielo (En mg N/100 g de muestra)

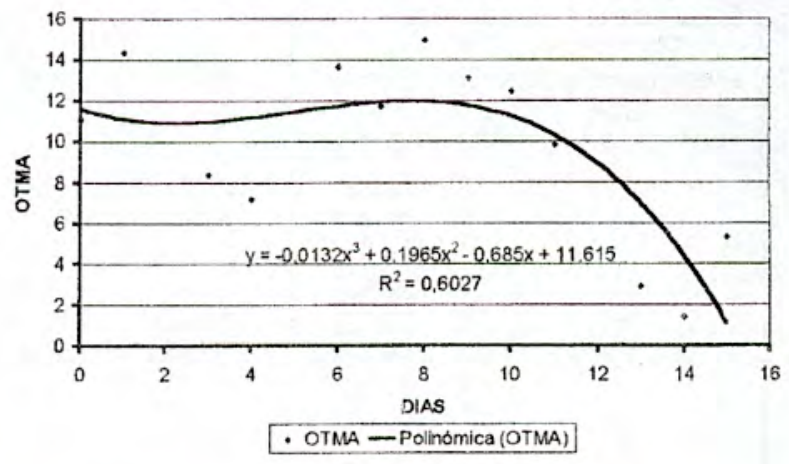

Figura 5. Contenido de TMA en el Lenguado de Profundidad almacenado en hielo (En $\mathrm{mg} \mathrm{N} / 100 \mathrm{~g}$ de muestra)

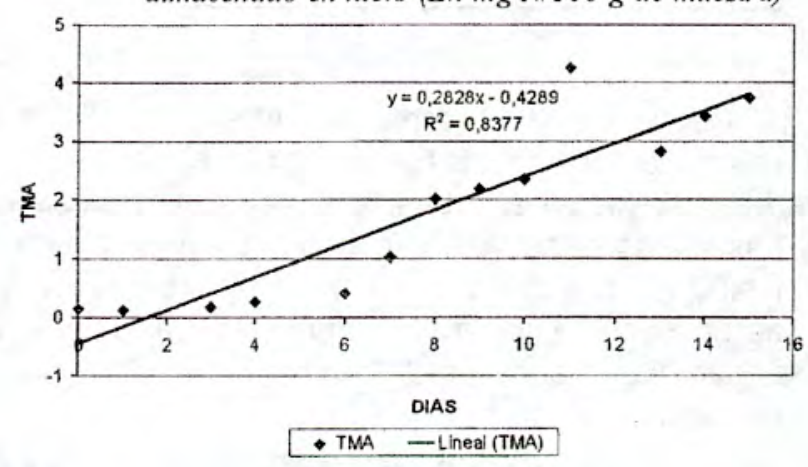

Figura 6. Contenido de TVN en el Lenguado de Profundidad almacenado en hielo (En mg N/100 g de muestra)

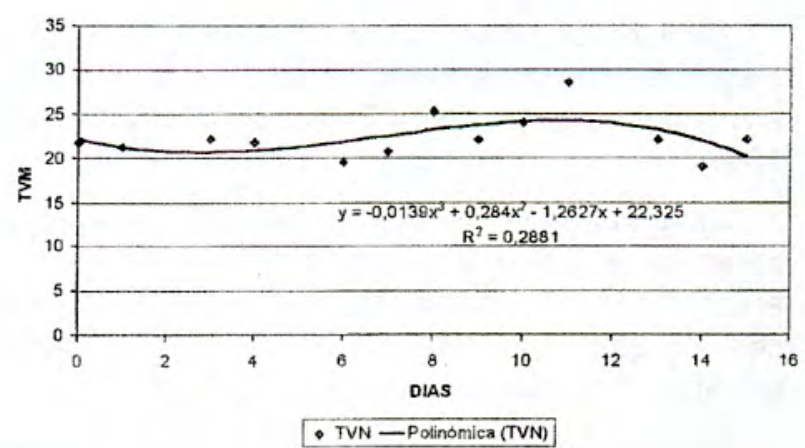

\subsection{Para el Sargo}

Los resultados obtenidos de los análisis de las determinaciones de OTMA, TMA y TVN, en las muestras de sargo fresco almacenado en hielo durante 15 dias, se presentan en la tabla N? 3 así como en las gráficas 7,8 y 9 . 
Cuadro 3. Contenido de OTMA, TMA y TVN en el Sargo fresco almacenado en hielo (En mg N/100 g de nuestra).

\begin{tabular}{|c|c|c|c|}
\hline $\begin{array}{c}\text { Tiempo de } \\
\text { almacenamiento en } \\
\text { dias }\end{array}$ & OTMA & TMA & TVN \\
\hline 0 & 26,72 & 0,18 & 19,32 \\
1 & 25,98 & 0,19 & 19,05 \\
2 & 26,41 & 0,21 & 20,47 \\
3 & 25,02 & 0,43 & 20,81 \\
4 & 23,83 & 0,64 & 19,92 \\
6 & 23,92 & 0,83 & 21,02 \\
7 & 23,78 & 1,15 & 21,28 \\
8 & 24,22 & 1,45 & 22,48 \\
9 & 22,91 & 1,65 & 23,03 \\
11 & 20,05 & 1,73 & 24,12 \\
13 & 16,75 & 1,96 & 25,80 \\
14 & 15,10 & 2,15 & 26,72 \\
15 & 13,56 & 2,26 & 27,02 \\
\hline
\end{tabular}

Según muestra la tabla, el contenido de OTMA al comenzar los análisis fue $26,72 \mathrm{mg}$. N/100 g; en los dias subsiguientes muestran un descenso paulatino hasta llegar al séptimo dia en que obtiene $23,78 \mathrm{mg}$. $\mathrm{N} / 100 \mathrm{~g}$. Sin embargo, a partir del octavo día se aprecia una bajada más pronunciada hasta llegar al 15 avo día en el cual el contenido de OTMA alcanzó un valor de $13,56 \mathrm{mg} \mathrm{N} / 100 \mathrm{~g}$.

En el caso de la TMA, el primer día los análisis arrojaron un resultado de $0,18 \mathrm{mg} \mathrm{N} / 100 \mathrm{~g}$; a los 7 dias se obtuvo $1,15 \mathrm{mg} \mathrm{N} / 100 \mathrm{~g}$, en los días subsiguientes.

También se observa un aumento paulatino, sin mayores distorsiones hasta llegar al quinceavo día, en el cual el contenido de TMA alcanza a 2,26 mgN/ $100 \mathrm{~g}$.

Observando la columna y gráfica $\mathrm{N}^{0} 9 \mathrm{del}$ TVN, apreciamos que durante los 15 días de almacenado el sargo en hielo, este indicador sufrió un incremento de $7,7 \mathrm{mgN} / 100$, el cual fue en una forma paulatina con ligeras desviaciones.

En lo referente a los cambios orgalépticos sufrido por el pescado durante los 15 días de almacenamiento, se pudo establecer que superficialmente cambió de color notándose los músculos con cierta elasticidad y olor a pescado bien acentuado, por lo que se considera que aun se podía consumir. También es preciso notar que estos cambios comienzan a ser notorios.
Figura 7. Contenido de OTMA en el Sargo fresco almacenado en hielo (En $\mathrm{mg} \mathrm{N} / 100 \mathrm{~g}$ de muestra)

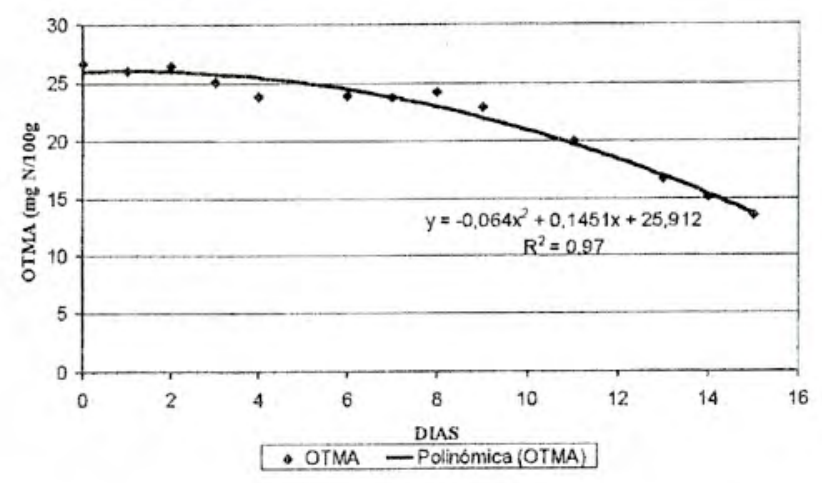

Figura 8. Contenido de TMA en el Sargo fresco almacenado en hielo (En mg N/100 $\mathrm{g}$ de muestra)

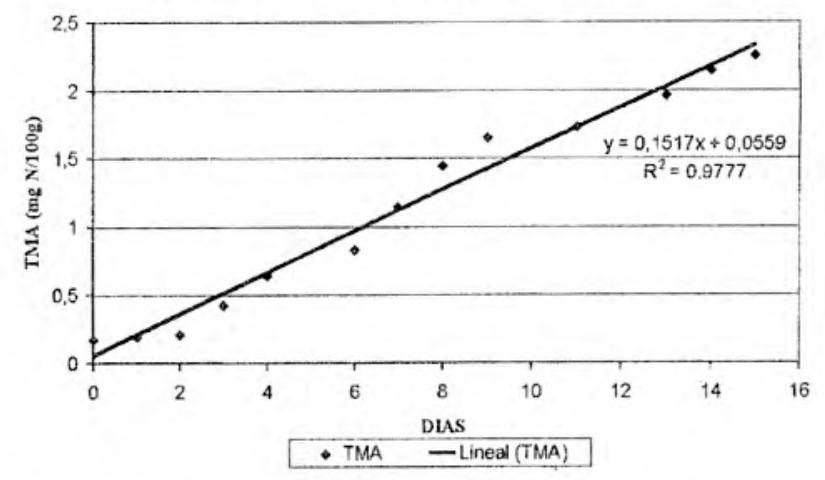

Figura 9. Contenido de TVN en el Sargo fresco almacenado en hielo (En mg N/100 g de muestra)

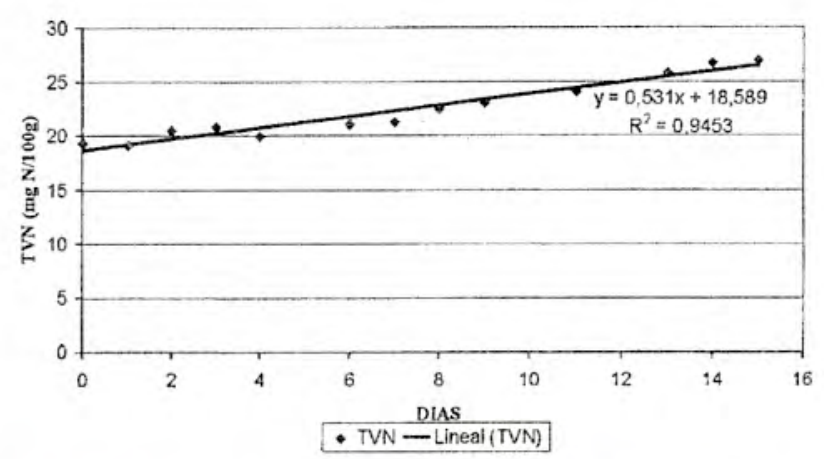

\section{CONCLUSIONES Y RECOMENDACIONES}

- Durante el almacenamiento en hielo del pescado fresco, se ha confirmado que los tres indicadores investigados difieren entre sí.

- Los cambios que se presentan durante el 
almacenamiento del lenguado son similares a los del bacalao, no sucede asi con los cambios que sufre el sargo en las mismas condiciones.

- Se ha podido confirmar que hay una estrecha relación entre el aumento del TMA y el empeoramiento de las cualidades organolépticas, las cuales se manifestaron más notoriamente después del sexto -octavo día de almacenamiento.

- En el bacalao de profundidad, entre el noveno y décimo día de almacenamiento, se presenta un significativo aumento en el contenido de TMA (de $4,18-15,55 \mathrm{mgN} / 100 \mathrm{~g}$ ), en el lenguado común este aumento se presenta entre el décimo y onceavo dia, (de 2,19 a $4,26 \mathrm{mgN} / 100 \mathrm{~g}$ ) y en el sargo entre el sexto y sétimo día de almacenamiento (de 0,8 - 1,15 $\mathrm{mgN} / 100 \mathrm{~g}$ ). Aunque dicho incremento no fue significativo. Es necesario indicar que durante el almacenamiento del lenguado y del sargo el aumento de TMA fue en forma regular, no así en caso del bacalao.

- Se recomienda hacer otras investigaciones con otros indicadores, ya que la TMA y el TVN, en la carne de las especies investigadas, recién se presentan entre el sexto y octavo dia por lo que su utilidad como indicadores objetivos de la calidad, al parecer, es problemática

\section{REFERENCIAS BIBLIOGRÁFICAS}

AGENCIA DE COOPERACIÓN INTERNACIONAL DEL JAPÓN. Métodos químicos de análisis; Pág. 19-21. Instituto Tecnológico Pesquero del Perú. 1998.

CHIRICHIGNO, F. Clave para identificar los peces marinos del Perú. Pág. 297. Instituto del Mar del Perú. Callao 1998.

CONTRERAS, E. Métodos de análisis químico para la harina de aceite de pescado: Pág. 46-47. Instituto de Fomento Pesquero de Chile. Santiago de Chile 1996.

HENRIK, H. El pescado fresco, su calidad y cambios de calldad. Organización de las Naciones Unidas para la Agricultura y la Alimentación, Organismo Danés de fomento Internacional. Roma, 1988.

IKEDA, S. Other Organic Components and Inorganic Components in advances in fish science and technslogy. Editado por J.J. connell, Fishing News Books Ltd. Surry, England, 1980.

INSTITUTO TECNOLÓGICO PESQUERO. XI Curso Internacional de Tecnológica de Procesamiento de Productos Pesqueros. Callao 1995.

KIKUCHI, T.; WADA, S. y SUZUKI, H. Significance of volatile bases and volatile acid in development of flavor of fish meat. J. Jap Soc. Fd. Murl, 29, 147 - 152, 1976.

FUJIWARA - ARASAKI T AAND. MINO. The distribution of $y$ trimethilamine and trimethilamina oxide in marine aiga. Proc: 7 ? Int. Seaweed sym (1971), Japan. Of Univ. 07 Tokio Press. $506-510$.

MEJIA, A. Pesca exploratoria del espinel de fondo para bacalao (Dissostichus eleginoides) a borde de la embarcación "Cangallo" del 11 al 20-12-96, informe, Centro de Entrenamiento Pesquero, Paita 1996.

MINISTERIO DE PESQUERÍA. Informe Estadístico del Comportamiento en la Pesca en el Perú. Información captada a través de INTERNET. Octubre de 1999.

SIKORSKI, Z.E. Technologia Zywnosci POCHODZENIA MORSKIEGO. Pag 163 - 169. Wyddwnicwo Naukowego. Warszdwa - Polonia 1993.

WATTS, R.L. y WATS, D.C. Nitrógeno Metabolismo in fishes, Chemical Zoology, Academic Press, London y New York. Vol $8,369-446,1974$ 